\title{
PERFORMANCE OF ANNULAR FLOW STERILIZER IRRADIATED BY A GERMICIDAL LAMP
}

\author{
TAKUo SUGAWARA, Michio YONEYA AND Hiroyasu OHASHI \\ Department of Chemical Engineering for Resources, \\ Akita University, Akita 010
}

\begin{abstract}
Theoretical and experimental studies were conducted on the UV-inactivation characteristics of Bacillus subtilis spores in an annular-flow sterilizer irradiated by a germicidal lamp located at the center of the inner tube.

Experimental results for sterilizer performance in laminar spore-suspension flow were well simulated dynamically and statically by theoretical considerations which incorporated multitargets with single-hit model for the UV-inactivation kinetic and the diffuse light model for the angle characteristic of lamp, along with due attention to the parabolic velocity distribution and the negligible diffusion of spores. Scale on the outside wall of the inner tube was checked by the use of a cylindrical chemical actinometer.

Calculated examples elucidate the reason why careful inspection is needed of UV-inactivation kinetics, angle characteristics of lamp, and fluid mixing to evaluate performance of actual flow UV sterilizers at high extent of inactivation up to the order of $99.999 \%$.
\end{abstract}

\section{Introduction}

In a line of research on sterilizers irradiated by a germicidal lamp (UV sterilizers), the authors ${ }^{9}$ previously reported the UV-inactivation kinetics of Bacillus subtilis spores, widely used for examining sterility, at high extent of sterilization (survival fraction down to $10^{-6}$ ) with the use of batch UV sterilizers. They indicated the applicability of multi-targets with single-hit model with consideration of a small portion of UV-resistant spores for the reasonable formulation of the kinetics in such a highly sterilized region. It is essential to estimate the absolute UV intensity and the effect of flow mechanics on spore inactivation to establish the design procedure of UV sterilizers in flow systems.

The authors studied the inactivation characteristics of Bacillus subtilis spores in an annular UV sterilizer in laminar flow to evaluate the effects of angle characteristic of a light source, scale on the quartz-sterilizer wall, and residence time distribution of fluid elements in any type of flow UV sterilizer.

\section{Experimental Apparatus and Procedure}

The schematic diagram of an annular UV sterilizer is shown in Fig. 1. The body of the sterilizer, E, consisted of an inner quartz tube with outside diameter of $92.3 \mathrm{~mm}$ and inside diameter of $86.3 \mathrm{~mm}$, and an outer Pyrex tube with inside diameter of $103.6 \mathrm{~mm}$. The light source, F, was a germicidal lamp, GL-30

\footnotetext{
Received February 12, 1981. Correspondence concerning this article should be addressed to T. Sugawara. M. Yoneya is with Government
} Industrial Research Institute, Tohoku, Sendai 983.
(Toshiba, 200V-30W, $26 \phi \times 868$ ), with the assigned illuminating length of $200 \mathrm{~mm}$ achieved by covering rest of tube with sheets of black paper. The lamp was fixed at the center of the inner quartz tube. The testsection length of the sterilizer was made to be $700 \mathrm{~mm}$ by covering the upper and lower part of the inside wall of the inner tube with sheets of black paper. Holder D was provided to avoid segregation of flow. Tubes, connectives and cocks were made of Teflon to minimize adhesion of spores. The whole apparatus was set in a sterilized room (number of falling germs was

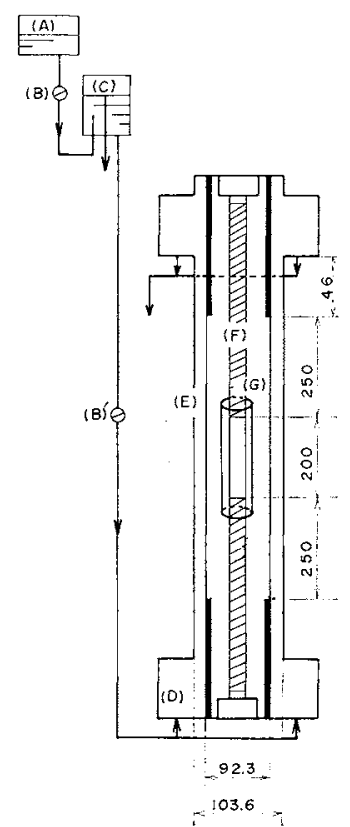

Fig. 1 Schematic diagram of annular UV-sterilizer 
less than 4-5 per hour).

The spore suspension of Bacillus subtilis NRRI with concentration of the order of $10^{2}$ to $10^{6} \mathrm{~cm}^{-3}$, was first stored in reservoir $\mathrm{A}$, then flowed through head tank $\mathrm{C}$ and entered holder D. Flowing up in an annulus, the suspension was irradiated in the test section by light source $F$, then overflowed to be measured volumetrically at each run. Flow rate was controlled by cock $\mathrm{B}^{\prime}$. The spore suspension, sampled at the exit, was quantitatively analyzed by counting the number of viable spores ${ }^{6}$. To determine the dynamic characteristics of the sterilizer, sampling was sequentially conducted after the suspension flow-rate became stationary and shutter $\mathrm{G}$, covering the illuminating part of a lamp, was removed. The details of cultivation of Bacillus subtilis NRRI and preparation of spore were described elsewhere ${ }^{6}$. Internal lightfiltering effect by spore suspension was checked by nephelometry and ascertained to be negligible under the experimental conditions studied.

\section{Absolute UV Intensity in an Annulus}

\section{1 Estimation by diffuse light model}

It is known that the radiant distribution characteristic is diffuse for the $254 \mathrm{~nm}$ resonance line of mercury vapor emitted from a germicidal lamp ${ }^{3}$. If the lamp is assumed to be a line source, the relative $\mathrm{UV}$ intensity at an arbitrary point, $\mathrm{P}(r, z)$, beneath the lamp of length $l$ can be represented by Eq. (1) ${ }^{1)}$ with the coordinates shown in Fig. 2:

$$
I(r, z) \propto \frac{1}{r}\left\{\frac{l-z}{\sqrt{(l-z)^{2}+r^{2}}}+\frac{z}{\sqrt{z^{2}+r^{2}}}\right\}
$$

Once we determine the absolute UV intensity with the use of a small vessel of chemical actinometer at the position of $\left(r_{s}, z_{s}\right)$ beneath the lamp of length $l_{s}$, we can calculate the absolute intensity at point $\mathrm{P}$ by Eq. (2):

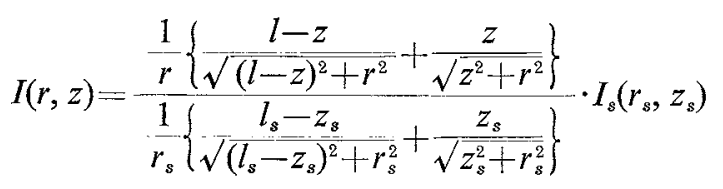

An example is as follows. For a watch-glass sterilizer placed $50 \mathrm{~mm}$ from a germicidal lamp with an illuminating length of $200 \mathrm{~mm}$, the experimental results are shown in Fig. 3 for the inactivation of Bacillus subtilis spores, the same microorganism as that used for the previous study ${ }^{9}$, along with the calculated line according to Eq. $\left.(3)^{9}\right)$ :

$$
f(\theta)=a_{1} \cdot f_{1}(\theta)+a_{2} \cdot f_{2}(\theta)
$$

where

$$
\begin{aligned}
& f_{j}(\theta)=1-\left[1-\exp \left\{-(\tilde{\phi \alpha})_{j} \cdot I \cdot \theta\right\}\right]^{m} \quad(j=1,2) \\
& a_{1}+a_{2}=1
\end{aligned}
$$

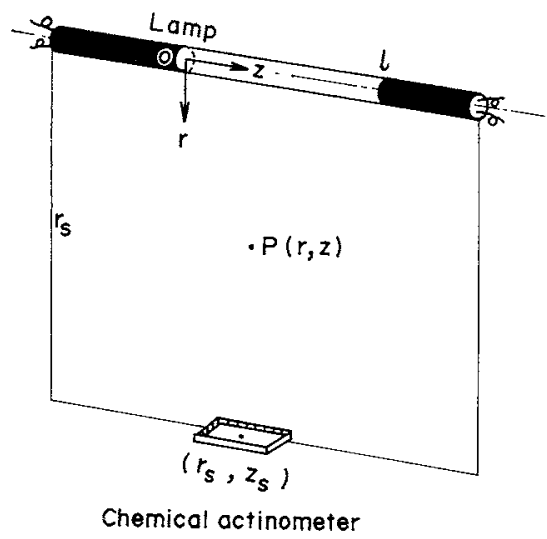

Fig. 2 Arrangement of light source and chemical actinometer to determine absolute UV intensity at $\mathrm{P}(r, z)$

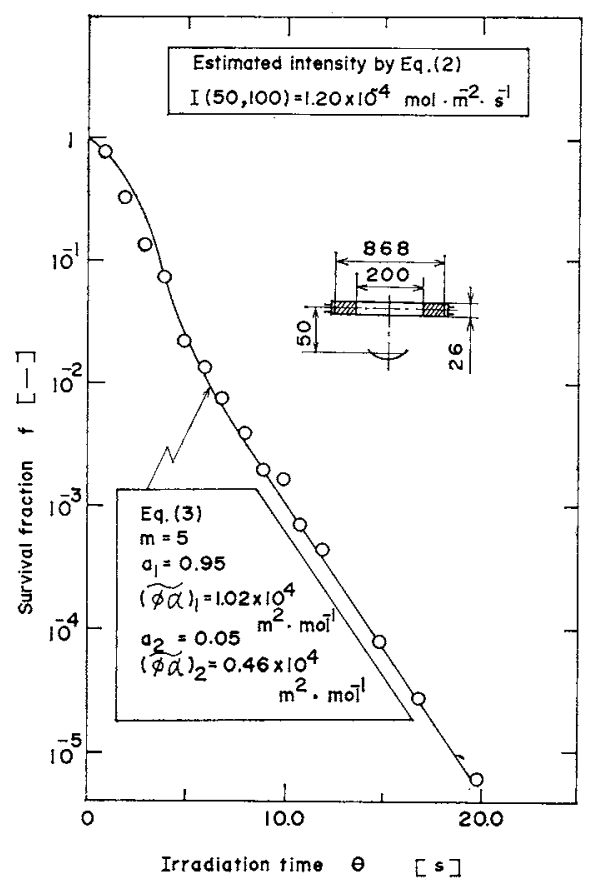

Fig. 3 Comparison of observed and calculated inactivation characteristics of Bacillus subtilis spores in a batch UV sterilizer; $I_{s}(500,434)=8.75$ $\times 10^{-8} \mathrm{~mol} \cdot \mathrm{m}^{-2} \cdot \mathrm{s}^{-1}\left(l_{s}=868\right)$

The absolute UV intensity, $I$, was calculated to be $1.20 \times 10^{-4} \mathrm{~mol} \cdot \mathrm{m}^{-2} \cdot \mathrm{s}^{-1}$ on the basis of the reference intensity, $I_{s}$, of $8.75 \times 10^{-8} \mathrm{~mol} \cdot \mathrm{m}^{-2} \cdot \mathrm{s}^{-1}$ at a point $500 \mathrm{~mm}$ beneath a germicidal lamp, GL-30, with an illuminating length of $868 \mathrm{~mm}$. The fractions of UVsensitive and UV-resistant spores, $a_{1}$ and $a_{2}$, the inactivation cross-sections, and target number are represented by Eqs. (6), (7) and (8) $)^{9}$.

$$
\begin{array}{ll}
a_{1}=0.95 & (\tilde{\phi \alpha})_{1}=1.02 \times 10^{4} \mathrm{~m}^{2} \cdot \mathrm{mol}^{-1} \\
a_{2}=0.05 & (\tilde{\phi \alpha})_{2}=0.46 \times 10^{4} \mathrm{~m}^{2} \cdot \mathrm{mol}^{-1} \\
m=5 &
\end{array}
$$

Observed values shown in Fig. 3 are in good agreement with theoretical values in which the effect of reflection of light on the surface was neglected. 


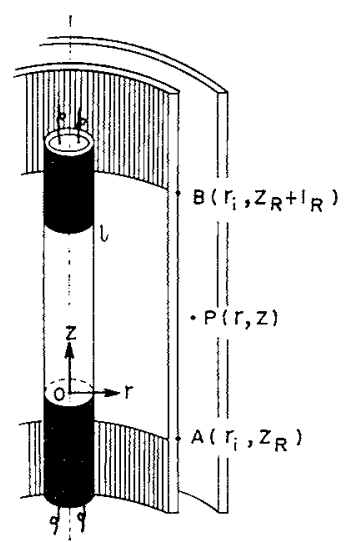

Fig. 4 Coordinates for estimation of absolute $\mathrm{UV}$ intensity at an arbitrary point, $\mathrm{P}(r, z)$, in an annulus

The absolute UV intensity at an arbitrary point $\mathrm{P}(r, z)$ in the annulus shown in Fig. 4 was estimated by Eq. (2) with correction of the scale on the sterilizer wall, as discussed in the following section, and neglecting reflection and absorption of light by the quartz glass.

\section{2 Measurement of UV intensity with a cylindrical} chemical actinometer and definition of cleanness factor

Incident intensity depression caused by the scale on the reactor wall is unavoidable for photoreactors in general $^{7}$. The authors determined the absolute UV intensity by inserting a cylindrical quartz-tube actinometer into the annulus after each run. The tube, with inside diameter of $3.3 \mathrm{~mm}$ and length of $200 \mathrm{~mm}$, contained potassium trioxalatoferrate (III) solution ${ }^{4}$.

The cleanness factor was defined as a measure of the filter effect of scale on the outside wall of the inner quartz tube by Eq. (9):

$$
\sigma=I^{\prime} / I_{o}^{\prime}
$$

where $I^{\prime}$ is absolute UV intensity in the annulus after a certain period of usage, and $I_{o}^{\prime}$ is that at the initial stage. The authors evaluated $I^{\prime}$ by Eq. (10), assuming incident beams to be parallel ${ }^{11}$ owing to the same length of actinometric tube as that of the illuminating section of the lamp, and the small diameter of actinometric tube $(3.3 \mathrm{~mm})$ compared with the large diameter of the lamp $(26 \mathrm{~mm})$ and the short distance between them $(49 \mathrm{~mm})$, and considering the effect of thickness of the cylindrical reactor wall, i.e. the effect of refraction ${ }^{8)}$, to be negligible for calculating UV intensity.

$$
I^{\prime}=\frac{(\pi / 4) d^{2}}{d \cdot \phi_{a}} \cdot \frac{d c_{R}}{d \theta}=\frac{\pi d}{4 \phi_{a}} \cdot \frac{c_{R}}{\theta}
$$

where $c_{R}$ is ferrous ion concentration produced by irradiation for $\theta, d$ is inside diameter of cylindrical tube, and $\phi_{a}$ is quantum yield for ferrous ion production $^{4}$.

\section{Theoretical Consideration of Annular UV Sterilizer Performance in Laminar Flow}

The velocity profile is represented for the fully developed laminar flow in an annulus by Eq. $(10)^{2}$.

$$
u(r)=2 \bar{u} \frac{\left\{1-\left(r / r_{o}\right)^{2}+\left(1-\kappa^{2}\right) \ln \left(r / r_{o}\right) / \ln (1 / \kappa)\right\}}{\left\{\left(1-\kappa^{4}\right) /\left(1-\kappa^{2}\right)-\left(1-\kappa^{2}\right) / \ln (1 / \kappa)\right\}}
$$

When the shape of Bacillus subtilis spore is assumed to be spherical with the diameter of ca. $1 \mu \mathrm{m}$, its diffusion coefficient is estimated to be of the order of $10^{-12} \mathrm{~m}^{2} \cdot \mathrm{s}^{-1}$; then the spore has the little probability of getting out of a certain fluid element. Consequently, it would be permissible to consider that each spore may move within a segregated fluid element. Under such a condition, the flow-averaged survival fraction for flowing suspension at the sterilizer exit, $f_{\mathrm{av}}$, can be given by Eq. (12):

$$
f_{\mathrm{av}}=\int_{r_{i}}^{r_{0}} 2 \pi r \cdot u(r) \cdot f(\theta, r) d r / \int_{r_{i}}^{r_{0}} 2 \pi r \cdot u(r) d r
$$

$f(\theta, r)$ is calculated by Eq. (13):

$$
f(\theta, r)=a_{1} \cdot f_{1}(\theta, r)+a_{2} \cdot f_{2}(\theta, r)
$$

where

$$
\begin{gathered}
f_{j}(\theta, r)=1-\left[1-\exp \left\{-(\tilde{\phi} \alpha)_{j} \cdot I_{s} \cdot \sigma \cdot \frac{\bar{I}(r)}{I_{s}} \cdot \tau(r)\right\}\right]^{m} \\
(j=1,2) \\
a_{1}+a_{2}=1 \\
\tau(r)= \begin{cases}\theta \quad(\theta<t(r)) \\
t(r)(\theta \geqq t(r))\end{cases} \\
t(r)=l_{R} / u(r)
\end{gathered}
$$

$\bar{I}(r)$ in Eq. (14), the longitudinally averaged UV intensity at radial point $r$, can be calculated by inserting Eq. (2) into Eq. (17).

$$
\frac{\vec{I}(r)}{I_{s}}=-\frac{1}{l_{p}} \int_{z_{p}}^{I_{R}+z_{R}} \frac{I(r, z)}{I_{s}} d z
$$

where

$$
\begin{aligned}
& l_{p}=\left\{\begin{array}{cc}
u(r) \cdot \theta & (\theta<t(r)) \\
l_{R} & (\theta \geqq t(r))
\end{array}\right. \\
& z_{p}=\left\{\begin{array}{cc}
z_{R}+l_{R}-u(r) \cdot \theta & (\theta<t(r)) \\
z_{R} & (\theta \geqq t(r))
\end{array}\right.
\end{aligned}
$$

$z_{R}$ is $z$-coordinate of the lower end of the test section, as seen in Fig. 4.

\section{Experimental Results and Discussion}

\section{1 Dynamic characteristics}

Figure 5 shows an example of the sequential relation of observed flow-averaged survival fraction, $f_{\mathrm{av}}$, at the sterilizer exit and elapsed time, $\theta$, after the shutter was removed, for Bacillus subtilis spores with the inactivation kinetic that $m$ equals $5 ; a_{1}$ and $(\tilde{\phi} \alpha)_{1}$ are 1.0 and $0.79 \times 10^{4} \mathrm{~m}^{2} \cdot \mathrm{mol}^{-1}$, respectively, under the condition that the initial spore concentration was $4.3 \times 10^{6} \mathrm{~cm}^{-3}$ and mean residence time was $43.2 \mathrm{~s}$. The reference UV intensity for the lamp, $I_{s}$, was $7.2 \times 10^{-6} \mathrm{~mol} \cdot \mathrm{m}^{-2} \cdot \mathrm{s}^{-1}$ at the position of $500 \mathrm{~mm}$ 


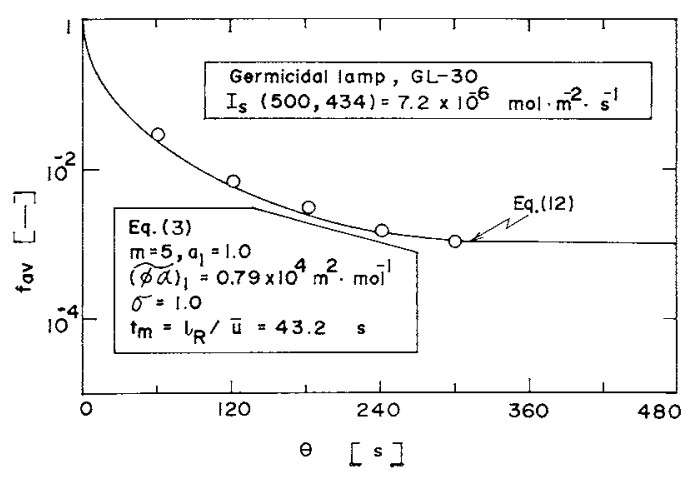

Fig. 5 Dynamic inactivation characteristic of Bacillus subtilis spores in an annular UV sterilizer in laminar flow

beneath the lamp with an illuminating length of $868 \mathrm{~mm}$. The cleanness factor of the sterilizer, $\sigma$, was 1.0. With the information mentioned above, theoretical values were calculated by Eq. (12) and are represented in the figure by the solid line. The theoretical line simulates the experimental results well. The figure indicates the period of unsteady state for about 300 seconds, considerably longer than the mean residence time because of longer residence time for spores flowing near the wall. It can be noted that the induction period for laminar-flow UV sterilizer is quite different from that for the plug-flow sterilizer at the extent of sterilization of $99.9 \%$, although the behavior of the laminar-flow photoreactor was reported to be rather similar to that of the plug-flow one at relatively low extent of conversion ${ }^{10)}$.

\section{2 Characteristics at steady state}

Examples of sterilizer performance at steady state are plotted in Fig. 6 for Bacillus subtilis spores with the inactivation kinetic represented by Eqs. (6), (7) and (8) under the condition of initial spore concentration on the order of $10^{3}$ to $10^{6} \mathrm{~cm}^{-3}$. The reference UV intensity for the lamp applied here was $8.75 \times$ $10^{-6} \mathrm{~mol} \cdot \mathrm{m}^{-2} \cdot \mathrm{s}^{-1}$ at a position of $500 \mathrm{~mm}$ beneath the lamp with an illuminating length of $868 \mathrm{~mm}$. The cleanness factor of the sterilizer wall was 0.71 . Theoretical performance was calculated at steady state $\left(\theta \gg t_{m}\right)$ by Eq. (12), and is represented by a thick solid line. The theoretical line simulates the experimental results well up to the high extent of sterilization of $99.999 \%$. The dotted line in Fig. 6 represents the calculated results, neglecting the fraction of UVresistant spores. It can be seen that the deviation of the dotted line from experimental data increases as the extent of sterilization becomes higher.

Flow-averaged survival fraction of Bacillus subtilis spores was calculated on the assumptions that the angle characteristic of light source was taken to be specular $^{1\rangle}$ and that the other conditions were the same as those for the case of the thick solid line in Fig. 6 . Calculated performance is shown as a solid line in

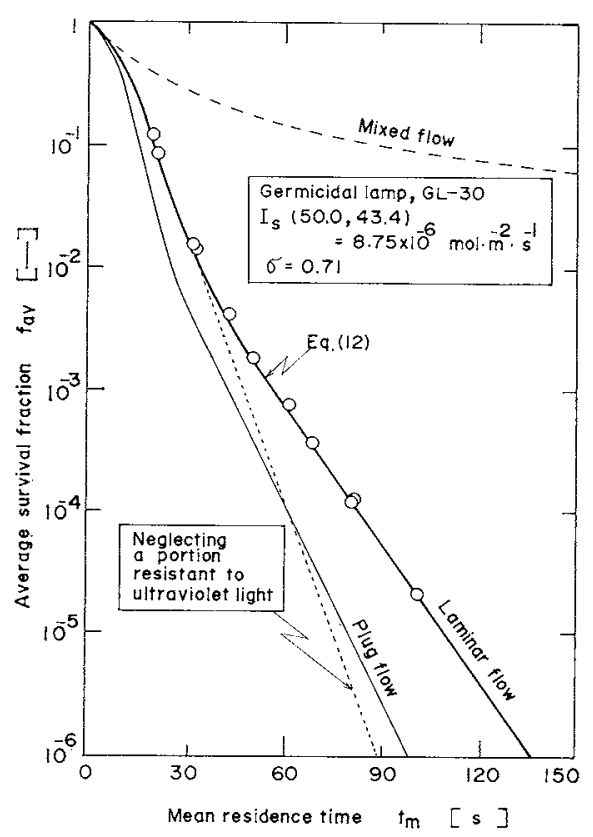

Fig. 6 Steady-state inactivation characteristics of Bacillus subtilis spores in annular flow UV sterilizers

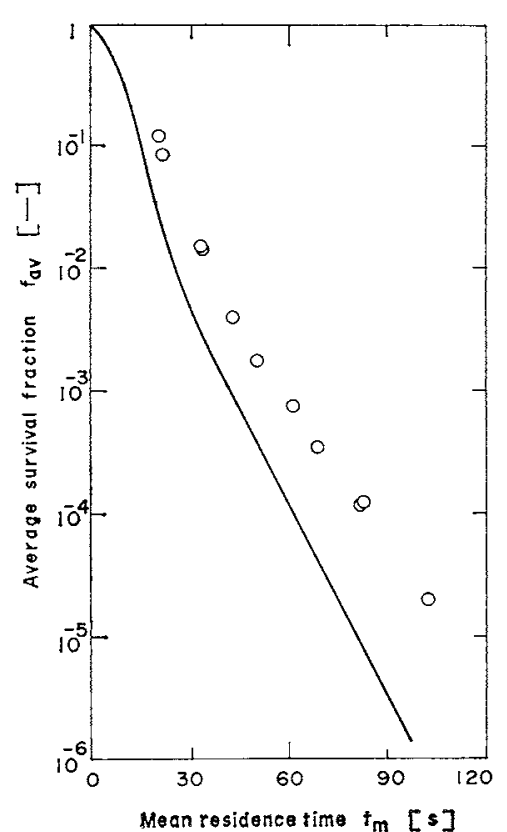

Fig. 7 Comparison of observed inactivation characteristics of Bacillus subtilis spores in an annular UV sterilizer in laminar flow with calculated curve assuming angle characteristic of lamp to be specular

Fig. 7. Extent of sterilization would be estimated too high in the high inactivation region by the specular light model even if the effect is relatively small at low extent of sterilization.

\section{3 Effect of fluid mixing on annular flow UV steri- lizers}

Theoretical performance was calculated for an annular plug-flow UV sterilizer of the same geometry as that used in the present study by Eq. (20): 


$$
f_{\mathrm{a} \nabla}\left(t_{m}\right)=\int_{0}^{\infty} f\left(\theta, \frac{r_{0}+\frac{t+r_{i}}{2}}{2}\right) \cdot \delta\left(\theta-t_{m}\right) d \theta=f\left(t_{m}, \frac{r_{o}+r_{i}}{2}\right)
$$

where $f\left(\theta, r_{o}+r_{i}\right) / 2$ was calculated by Eq. (13); the radial distance of $\left(r_{o}+r_{i}\right) / 2$ (the center of annulus) was chosen to evaluate the light intensity distribution in the annular plug-flow sterilizer. The result is shown in Fig. 6 by a thin solid line. A broken line in the figure also represents the theoretical characteristic, calculated by Eq. (21), for a macrofluid-type mixed-flow ${ }^{57}$ UV sterilizer of the same geometry:

$$
f_{\mathrm{av}}\left(t_{m}\right)=\frac{1}{t_{m}} \int_{0}^{\infty} f\left(\theta, \frac{r_{o}+r_{i}}{2}\right) \cdot \exp \left(-\theta / t_{m}\right) d \theta
$$

The broken line indicates the lowest efficiency in sterilizer performance: youngest fluid elements leaving the mixed-flow sterilizer affect more as the extent of inactivation gets higher. The characteristics for these two extreme flow conditions suggest that it would generally be difficult to obtain a high extent of sterilization in actual flow UV sterilizers having shortcircuit channels; it is preferable to use plug-flow types for achieving high inactivation.

\section{Conclusion}

Dynamic and steady-state performance was determined for the laminar suspension flow of Bacillus subtilis NRRI spores in an annular sterilizer irradiated by a germicidal lamp located at the center of the inner quartz tube. Experimental results were simulated well by the improved one-hit-killing model for the inactivation kinetic and by the diffuse light model for the angle characteristic of lamp, along with consideration of the scale on the sterilizer wall and the velocity distribution.

Extent of sterilization would be overestimated in the high-sterility region if the angle characteristic of light source were taken to be specular. It is difficult to achieve a high extent of inactivation with the use of flow sterilizers having short-circuit channels. It is best to apply a plug-flow UV sterilizer, and also advisable to use a laminar-flow sterilizer at steady state in that the flow pattern is distinct.

\section{Acknowledgment}

The authors are most grateful to Dr. Shigenori Tamagawa, Director of the Clinical Laboratory at Tokyo Metropolitan Komagome Hospital, for information on Bacillus subtilis NRRI. They also appreciate the experimental assistance of Mr. Y. Tanji (now with Nihon Kokan, KK) and Miss S. Kurosawa. This work was supported by the Asahi Glass Foundation for Industrial Technology in 1977 and 1978, and by a Grant-in-Aid for Scientific Research from the Ministry of Education, Science and Culture, Japan in 1978 (No. D365341).

* We can also derive Eq. (20) from Eq. (12).

\section{Nomenclature}

$=$ constant

$c_{R} \quad=$ ferrous ion concentration

$d \quad=$ inside diameter of cylindrical actinometric tube

$=$ survival fraction of spores

$=$ light intensity

$=$ length of light source

$=$ defined by Eq. (18)

$=$ test-section length

$=$ target number

$=$ radial distance from center-line of light source

$=$ outside radius of inner tube

$=$ inside radius of outer tube

$=l_{R} / u$

$r_{0}$

$t_{m}$

$t_{m}$
$u$

$u$

$\bar{u}$

$z$

$z^{2}$

$z_{p}$

$z_{R}$

$\alpha$

$\delta$

$\theta$

$\kappa$

$\sigma$

$\tau$

$\phi$

$\phi_{a}$

$\langle$ Subscripts〉

$$
\begin{array}{ll}
\text { av } & =\text { averaged value for flowing suspension } \\
j & =1: \text { UV-sensitive; }=2 \text { : UV-resistant } \\
o & =\text { initial stage or inside of outer tube } \\
s & =\text { reference point or reference length } \\
\lambda & =\text { wavelength }
\end{array}
$$

$\langle$ Superscripts〉

$$
\begin{array}{ll}
\sim & =\text { averaged value for wavelength } \\
- & =\text { averaged value for sterilizer length } \\
& =\text { actinometric study }
\end{array}
$$

\section{Literature Cited}

1) Akehata, T. and T. Shirai: J. Chem. Eng. Japan, 5, 385 (1972).

2) Bird, R. B., W. E. Stewart and E. N. Lightfoot: "Transport Phenomena", p. 51, John Wiley and Sons, New York (1960).

3) Funayama, H., K. Ogiwara, T. Sugawara and H. Ohashi: Kagaku Kogaku Ronbunshu, 3, 345 (1977).

4) Ito, M. ed.: "Shin Jikken Kagaku Koza, Kiso Gijutsu 3, Hikari I", p. 241, Maruzen, Tokyo (1976).

5) Levenspiel, O.: "Chemical Reaction Engineering, 2nd ed.", p. 329, John Wiley and Sons, New York (1972).

6) Ohashi, H., T. Sugawara, H. Kondo and T. Suzuki: Reports of the Asahi Glass Foundation for Industrial Technology, 35, 63 (1979).

7) Sugawara, T., H. Ohashi and T. Tadaki: Kagaku Kogaku Ronbunshu, 1, 160 (1975). 
8) Sugawara, T., M. Yoneya and H. Ohashi: Preprint of 44th Annual Meeting of The Soc. Chem. Engrs., Japan, A208 (Tokyo, April, 1979).

9) Sugawara, T., M. Yoneya, H. Ohashi and S. Tamagawa: J. Chem. Eng. Japan, 14, 400 (1981).

10) Yamaguchi, M., T. Sugawara and H. Ohashi: Preprint of
Gunma Meeting of The Soc. Chem. Engrs., Japan, A18 (Kiryu, July, 1978).

(Presented in part at 13th Autumnal Meeting of The Soc. of Chem. Engrs., Japan, SI206, Nagoya, October, 1979.)

\title{
Short Communications
}

\section{DENSITIES,VISCOSITIES AND AMINE DIFFUSIVITIES OF AQUEOUS MIPA, DIPA, DGA AND EDA SOLUTIONS}

\author{
Haruo HIKITA, Haruo ISHIKAWA, Tetsuya MURAKAMI and Tohru ISHII \\ Department of Chemical Engineering, University of Osaka Prefecture, Sakai 591
}

Aqueous solutions of amines are frequently used for the removal of acidic gases from gas mixtures. Therefore, a knowledge of the diffusivities of amines in aqueous solutions of various concentrations is important in the rational design of absorption equipment. In our previous work ${ }^{2)}$, the diffusivities of MEA (monoethanolamine), DEA (diethanolamine) and TEA (triethanolamine) in aqueous solutions were measured and these measured diffusivities were found to be considerably well correlated by the empirical relation, $D / D_{w}=\left(\mu / \mu_{w}\right)^{-2 / 3}$.

In the present study, the diffusivities of MIPA (monoisopropanolamine), DIPA(diisopropanolamine), DGA (diglycolamine) and EDA (ethylenediamine) in aqueous solutions, together with the densities and viscosities of these amine solutions, were measured and compared with the above empirical relation.

\section{Experimental}

The diffusivities of MIPA, DIPA, DGA and EDA in aqueous solutions were measured by the diaphragm cell technique described by Stokes ${ }^{5}$. The experimental apparatus and procedure were essentially the same as those in our previous work ${ }^{2}$.

The densities of the aqueous amine solutions were measured by means of a $25 \mathrm{~cm}^{3}$ calibrated pycnometer, and the viscosities of the solutions were determined with an Ostwald viscometer with efflux times of several hundred seconds.

All the measurements were carried out at $25^{\circ} \mathrm{C}$ and atmospheric pressure.

Received January 26,1981 . Correspondence concerning this article should be addressed to H. Hikita

\section{Results and Discussion}

The measured values of the densities $\rho$ and viscosities $\mu$ of the aqueous solutions of MIPA, DIPA, DGA and EDA are given in Table 1 as functions of amine concentration $C$. The estimated accuracies in the density and viscosity measurements are $0.01 \%$ and $0.2 \%$, respectively. The viscosity data of Sada et al. ${ }^{3)}$ obtained at $25^{\circ} \mathrm{C}$ for aqueous MIPA and DIPA solutions are in good agreement with the present

\begin{tabular}{|c|c|c|c|}
\hline Amine & $\begin{array}{c}\text { Conc. of } \\
\text { amines } \\
C\left[\mathrm{kmol} / \mathrm{m}^{3}\right]\end{array}$ & $\begin{array}{l}\text { Density } \\
\rho\left[\mathrm{kg} / \mathrm{m}^{3}\right]\end{array}$ & $\begin{array}{l}\text { Viscosity } \\
\mu[\mathrm{mPa} \cdot \mathrm{s}]\end{array}$ \\
\hline MIPA & $\begin{array}{l}0.199 \\
0.495 \\
1.65 \\
2.58 \\
3.69\end{array}$ & $\begin{array}{r}997 \\
997 \\
998 \\
999 \\
1002\end{array}$ & $\begin{array}{l}0.943 \\
1.021 \\
1.417 \\
1.905 \\
2.774\end{array}$ \\
\hline DIPA & $\begin{array}{l}0.497 \\
0.902 \\
1.75 \\
2.13 \\
2.78 \\
3.17 \\
3.53\end{array}$ & $\begin{array}{l}1001 \\
1004 \\
1012 \\
1016 \\
1022 \\
1024 \\
1028\end{array}$ & $\begin{array}{c}1.180 \\
1.521 \\
2.752 \\
3.682 \\
6.281 \\
8.872 \\
12.40\end{array}$ \\
\hline DGA & $\begin{array}{l}0.485 \\
0.985 \\
2.06 \\
2.86\end{array}$ & $\begin{array}{l}1002 \\
1006 \\
1018 \\
1026\end{array}$ & $\begin{array}{l}1.044 \\
1.234 \\
1.826 \\
2.504\end{array}$ \\
\hline EDA & $\begin{array}{l}0.501 \\
1.00 \\
1.54 \\
2.50 \\
3.60\end{array}$ & $\begin{array}{l}996 \\
995 \\
994 \\
993 \\
993\end{array}$ & $\begin{array}{l}0.994 \\
1.107 \\
1.248 \\
1.581 \\
2.112\end{array}$ \\
\hline
\end{tabular}

